\title{
An evidence-based approach to positive sentinel node disease: should we ever do a completion node dissection?
}

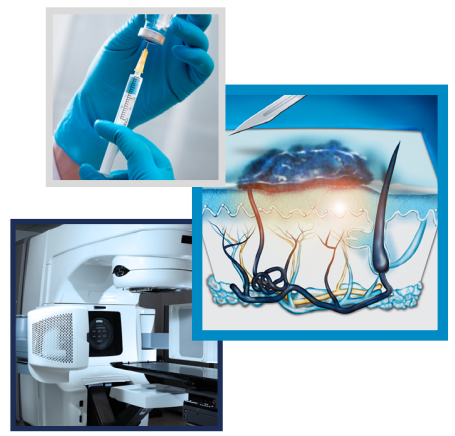

\author{
Jennifer S Downs*, (iD) \& David E Gyorki ${ }^{1,2,3}$ \\ ${ }^{1}$ Division of Cancer Surgery, Peter MacCallum Cancer Centre, Melbourne, Victoria, 3000, Australia \\ ${ }^{2}$ Department of Surgery, University of Melbourne, Melbourne, Victoria, 3000, Australia \\ ${ }^{3}$ Victorian Comprehensive Cancer Centre, Melbourne, Victoria, 3000, Australia \\ *Author for correspondence: jennifer.downs@petermac.org
}

\begin{abstract}
Management of later stage melanoma has undergone significant changes. Sentinel node biopsy has long been an accepted method of staging, but two recent randomized-controlled trials have provided an evidence base for decision making about completion lymphadenectomy. They showed no survival advantage in further surgery for patients with positive sentinel node biopsies. There is now no evidence to support completion lymphadenectomy in the majority of patients, and this is reflected in international practice guidelines.
\end{abstract}

First draft submitted: 29 March 2019; Accepted for publication: 6 June 2019; Published online: 18 October 2019

Keywords: DeCOG-SLT • lymph node • lymphadenectomy • melanoma • MSLT-2 • sentinel node

Melanoma has long been a disease cured by the knife. Since first mentioned by Hunter, Laennec, Cooper and other published surgeons of the 18th and 19th centuries, the only hope for prolonged survival for most patients with a diagnosis of melanoma was to completely excise all evidence of tumor prior to metastasis [1]. As early as 1892, surgeons were advocating that lymphadenectomy should be performed in an attempt to remove all traces of the cancer [2].

However, lymphadenectomy is not without complications. A systematic review of recent literature (2000-2017) showed that therapeutic lymphadenectomy has a reported complication rate of up to 39\%, including wound infection, wound breakdown, lymphedema and seroma [3]. Lymphedema has a significant impact on patients' quality of life.

Prior to the widespread adoption of sentinel node biopsy (SNB), a number of randomized-controlled trials were conducted comparing elective lymph node dissection (ELND) with observation $[4,5]$. While none of these demonstrated a survival advantage to elective lymph node dissection, a subgroup of patients was identified with an improved outcome from early surgical resection of the lymph node basin - intermediate thickness lesions with no clinically evident nodal disease. However, the same trials also showed that on average, only approximately 15-25\% of patients had tumor detected in the lymphadenectomy specimen $[6,7]$.

\section{Sentinel node biopsy}

Since 1992, the work of Morton and others on the sentinel lymph node has provided surgeons with a less morbid modality for accurate lymph node staging [8]. In patients with clinically node negative melanoma, SNB provides a more accurate assessment of the lymph node status (by providing low volume tissue to pathology allowing thorough sampling and assessment) and with much lower morbidity, with a lymphedema rate of only $1.3-6.3 \%[3,9,10]$. SNB has repeatedly been validated as an acceptable method to diagnose the presence of clinically occult lymph node disease, and provide accurate $\mathrm{N}$ staging [11].

For patients with tumor-positive nodes, MSLT-1 demonstrated a survival benefit for those patients in the SNB arm compared with those who underwent delayed lymphadenectomy after a period of nodal observation and therapeutic lymphadenectomy when required. The nodal tumor burden in the SNB group was much smaller and

Future $\because$ Medicine 
Table 1. Comparison of MSLT-2 and DeCOG-SLT studies.

\begin{tabular}{|c|c|c|c|c|}
\hline Results & & SLT-2 & & DG-SLT \\
\hline Number of patients analyzed & 1755 & & 473 & \\
\hline Sites of primary disease & All & & Trunk, arms, le & \\
\hline Sentinel node tumor burden & & & & \\
\hline$-<1.0 \mathrm{~mm}$ & $786(50 \%)$ & & $311(65 \%)$ & \\
\hline$->1.0 \mathrm{~mm}$ & $403(26 \%)$ & & $121(26 \%)$ & \\
\hline Not specified & $375(24 \%)$ & & $41(9 \%)$ & \\
\hline $\begin{array}{l}\text { Positive lymph nodes identified } \\
\text { at immediate completion } \\
\text { lymph node dissection }\end{array}$ & $11.5 \%$ & & $18 \%(25 \%$ dat & \\
\hline Median follow-up & 43 months & & 35 months & \\
\hline Reported at 3 years & Dissection & Observation & Dissection & Observation \\
\hline Recurrence-free survival & $68 \%+-1.7^{\dagger}$ & $63 \%+-1.7^{\dagger}$ & $66.8 \%+-5.9$ & $67.4 \%+-5.8$ \\
\hline Distant metastasis-free survival & $\sim 73 \%^{\ddagger}$ & $\sim 73 \%^{\ddagger}$ & $75 \%+-5.1$ & $78 \%+-5.4$ \\
\hline Melanoma-specific survival & $86 \%+-1.3$ & $86 \%+-1.2$ & $81.2 \%+-5.1$ & $81.7 \%+-4.9$ \\
\hline
\end{tabular}

the rate of nodal involvement at completion lymph node dissection (CLND) was only approximately $11 \%$ [9]. As such, the majority of patients underwent this procedure for no clinical benefit.

MSLT-1 confirmed that sentinel node status is the most important prognostic factor in staging intermediate thickness melanomas. In patients with clinically lymph node-negative melanoma, it is the best single predictor of melanoma-specific survival. This change in the understanding of how nodal disease affects prognosis is reflected in the eighth edition of the American Joint Committee on Cancer (AJCC) melanoma staging [7]. In this most recent staging system based on prospectively collected data from over 40,000 patients, an accurate stage cannot be allocated to a patient with melanoma $>1.0 \mathrm{~mm}$ in the absence of the results of Sentinel Node Biopsy (SNB). The eighth edition substratifies nodal disease according to clinically occult or clinically evident disease, number of involved nodes and presence of in-transit or satellite metastases. This increased detail allows for clearer prognostication within a heterogeneous group of patients [7]. There is a significant difference in 10-year melanoma-specific survival (MSS) depending on the number of involved nodes and their clinical status.

Important prognostic information is also obtained from the presence of disease within nonsentinel nodes. A single center retrospective review in fact identified this as the single most important predictor of mortality [12]. Mathematically designed scoring systems using both tumor and nodal characteristics (Non-Sentinel Node Risk Score [N-SNORE], other nomograms, the Rotterdam Criteria) have been validated in both internal and external cohorts to predict whether or not there will be nonsentinel lymph nodes found on completion lymphadenectomy, which can be used to individualize further treatment [13-16].

\section{Completion lymphadenectomy}

Given the low number of positive nonsentinel nodes found on completion lymphadenectomy in MSLT-1, and the lack of a strong rationale or evidence base for routine CLND, the MSLT-2 [9] study and the DeCOG-SLT [17] study were designed to answer the question of whether close surveillance of the lymph node basin with lymphadenectomy at the time of progression could provide equivalent outcome to immediate CLND in patients with a positive SNB (Table 1).

The MSLT-2 study was an international multicenter study, which prospectively randomized 1939 patients with positive sentinel nodes to either immediate CLND within 140 days or regular clinical and ultrasound surveillance of the relevant nodal basin. Patients were stratified for Breslow thickness, ulceration, method of metastasis detection and enrollment at an MSLT-1 center. They were healthy adults who were similar across demographics and other parameters including tumor and adjuvant therapy. The primary end point of the study was MSS. The majority of positive SNB contained deposits $<1 \mathrm{~mm}$ in size $(66 \%)$. Although there were patients in the dissection group who had nonsentinel nodes that were positive, and $26 \%$ of the observation group eventually went on to develop nodal disease, the early removal of possibly disease carrying nodes showed no improvement in MSS. However, completion lymphadenectomy did provide two positive outcomes. It allowed for diagnosis of non-sentinel node involvement, and and therefore more definitive prognostication. It also showed improved nodal recurrence-free survival [9]. 
DeCOG-SLT was a prospective randomized controlled trial run across multiple centers in Germany at a similar time period. However, they excluded head and neck melanomas from their trial group. The primary end point of the study was distant metastasis-free survival. Not only did the DeCOG-SLT study demonstrate no difference in MSS, it also showed no difference in recurrence-free survival. This is surprising as it would be expected that there would be a higher rate of recurrence in the observation arm given that a proportion have residual disease within the lymph node basin. The lack of difference in recurrence-free survival highlights the high risk of developing synchronous distant disease for patients with lymph node recurrence. In fact, while the observation arm identified lymph node recurrence in $25 \%$ of patients, only $7 \%$ of patients underwent CLND [17].

The conclusion of the DeCOG-SLT study was to not recommend CLND in patients with positive SNB with deposits of $1 \mathrm{~mm}$ or less [17]. The trial was closed early due to a lower than expected event rate, as well as a struggle to accrue randomized patients. MSLT-2 had similar accrual problems. This should not limit the applicability of their data. Rather, it is reflective of a global problem in recruiting patients into randomized surgical trials [17].

Other retrospective reviews have also shown similar results to these randomized trials, supporting the conclusion that there is limited role for immediate completion lymphadenectomy [18] given the minimal benefit and significant associated morbidity.

\section{Discussion}

These studies have been practice-changing and immediate CLND is no longer considered standard of care. The current Australian guidelines now state that 'CLND is no longer the preferred treatment for patients with a positive SLNB' [19]. Similarly, the current National Comprehensive Cancer Network (NCCN) guidelines offer the option of nodal observation or CLND but clarify that there is no evidence for improved survival, and that the improvement in regional control comes at a cost of increased morbidity [20].

This leads to the question of whether there is ever a role for elective CLND. There are three groups in whom immediate CLND ought to be considered. The first is patients undergoing SNB for a head and neck primary melanoma. These patients were under-represented in MSLT-2 and excluded from DeCOG-SLT, so the relevance of these trials in head and neck primaries is debatable. Furthermore, in the MSLT-2 study, the only factor that demonstrated a trend favoring immediate CLND was a head and neck primary and sentinel node [9]. Finally, the long-term morbidity of neck dissection is generally less than for axillary and ilio-inguinal lymphadenectomy, as it does not carry the same risks of lymphedema.

The second group is of patients who are unable or unwilling to participate in a surveillance protocol. The demonstration of equivalent outcomes with close observation and delayed lymphadenectomy at the time of progression relied on regular high-quality ultrasound surveillance. If these facilities cannot be provided, then an immediate CLND should be considered.

Finally, the information obtained from an immediate CLND can be of prognostic significance. The identification of further involved lymph nodes can upstage a patient from stage IIIA to IIIB or from IIIB to IIIC. However, a recent retrospective review of a European cohort of patients found that only approximately $6 \%$ of patients were upstaged following CLND. They also noted that stratifying these patients according to a model based on ulceration of the primary and sentinel node tumor burden provided equally good prognostication [21] and therefore the vast majority of patients will not receive added staging information from an immediate completion lymphadenectomy.

Consideration of CLND can also be given to those cases with high-risk features, as defined in the American Society of Clinical Oncology/Society of Surgical Oncology (ASCO/SSO) guidelines and excluded from MSLT-2. These features include extracapsular extension from the SNB, more than three positive lymph nodes, satellitosis of the primary tumor, and multiple or no identified draining nodal basins at the time of surgery [22]. However, all of the clinicopathological features that are predictors of nonsentinel node involvement are also predictors of distant metastases, and therefore it is difficult to define a population for whom immediate CLND should be recommended. For patients with these high-risk features the pros and cons of CLND should be discussed.

Adjuvant therapy is now offered in many parts of the world to patients who are SNB positive. Most trials of adjuvant therapy following SNB mandated an immediate CLND prior to enrollment. However, there is now consensus within the field that CLND is no longer required for the purpose of receiving adjuvant therapy [23]. In the last decade, the number of effective agents for adjuvant therapy of stage III and IV melanoma has expanded. Targeted therapy aimed at $B R A F$ gene mutations and checkpoint blockade immunotherapies have both been shown to be effective in the adjuvant setting. However, they are not without cost, both in terms of financial costs and adverse events (including mortality) caused by the drugs [24]. 


\section{Conclusion}

The last decade has seen a seismic transformation of the treatment landscape for patients with advanced melanoma. Effective adjuvant therapies (reviewed elsewhere in this series) are likely to have major impact on the survival for patients with stage-III disease. In this setting, the main role of SNB is now to provide risk stratification to identify patients who would benefit from these therapies. With contemporary adjuvant therapy, the vast majority of patients with positive SNB will not recur (either in the nodal basin or elsewhere). Therefore, the space for routine immediate CLND is now minimal.

\section{Future perspective}

The last few years have seen a dramatic evolution in the management of patients with sentinel node-positive melanoma. High-quality trials have led to a reduction in the need for regional therapy and associated morbidity with an increase in life-prolonging systemic therapy. However, these systemic therapies are not without their own morbidities and costs. The challenge in the management of sentinel node-positive melanoma is now in the identification of biomarkers that can identify those patients who despite nodal involvement are at low risk of disease progression and therefore can forego systemic therapy. Developing validated tools using gene expression profiling as well as markers of immune engagement may help to provide granular risk stratification to inform patient decision making.

\section{Executive summary}

\section{Sentinel node biopsy}

- Sentinel node biopsy (SNB) is required to stage all intermediate thickness melanoma, and SNB positivity is the single most important prognostic factor in melanoma staging.

- Non-SNB positivity also provides excellent prognostication, but very few patients studied would be upstaged by performing completion lymph node dissection (CLND) purely for staging purposes.

Completion lymphadenectomy

- MSLT-2 and DeCOG-SLT do not show any improvement in melanoma-specific survival or distant metastasis-free survival for patients who undergo immediate CLND.

- Current immunotherapy has further decreased the place for CLND.

Discussion

- CLND can be considered in high-risk subgroups: those not amenable to ongoing observation, head and neck melanoma, and high-risk features such as extracapsular extension or multiple positive SNBs.

Conclusion

- Routine immediate CLND has few current indications.

\section{Financial \& competing interests disclosure}

The authors have no relevant affiliations or financial involvement with any organization or entity with a financial interest in or financial conflict with the subject matter or materials discussed in the manuscript. This includes employment, consultancies, honoraria, stock ownership or options, expert testimony, grants or patents received or pending, or royalties.

No writing assistance was utilized in the production of this manuscript.

\section{Open access}

This work is licensed under the Attribution-NonCommercial-NoDerivatives 4.0 Unported License. To view a copy of this license, visit http://creativecommons.org/licenses/by-nc-nd/4.0/

\section{References}

Papers of special note have been highlighted as: $\bullet \bullet$ of considerable interest

1. Vito R, Sondak V, Smalley K. A brief history of melanoma: from mummies to mutations. Melanoma Res. 22(2), 114-122 (2013).

2. Snow H. Abstract of a lecture on melanotic cancerous disease. Lancet 140(3607), 872-874 (1892).

3. Moody JA, Botham SJ, Dahill KE, Wallace DL, Hardwicke JT. Complications following completion lymphadenectomy versus therapeutic lymphadenectomy for melanoma - a systematic review of the literature. Eur. J. Surg. Oncol.43(9), 1760-1767 (2017).

4. Balch CM, Soong SJ, Ross MI et al. Long-term results of a multi-institutional randomized trial comparing prognostic factors and surgical results for intermediate thickness melanomas (1.0 to $4.0 \mathrm{~mm}$ ). Ann. Surg. Oncol. 7(2), 87-97 (2000). 
5. Cascinelli N, Morabito A, Santinami M, Mackie RM, Belli F. Immediate or delayed dissection of regional nodes in patients with melanoma of the trunk: a randomised trial. Lancet 351(9105), 793-796 (1998).

6. Balch CM. The role of elective lymph node dissection in melanoma: rationale, results, and controversies. J. Clin. Oncol. 6(1), 163-172 (1988).

7. Gershenwald JE, Scolyer RA, Hess KR et al. Melanoma staging: evidence-based changes in the American Joint Committee on Cancer eighth edition cancer staging manual. CA. Cancer J. Clin. 67(3), 245-253 (2017).

8. Morton DL, Wen D-R, Wong JH et al. Technical details of intraoperative lymphatic mapping for early stage melanoma. Arch. Surg. 127(4), 392-399 (1992).

9. Faries MB, Thompson JF, Cochran $\mathrm{AJ}$ et al. Completion dissection or observation for sentinel-node metastasis in melanoma. N. Engl. J. Med. 376(23), 2211-2222 (2017).

-• The original paper from a large randomized controlled trial giving evidence for stopping immediate CLND.

10. Roaten JB, Pearlman N, Gonzalez R, Gonzalez R, McCarter MD. Identifying risk factors for complications following sentinel lymph node biopsy for melanoma. Arch. Surg.140(1), 85-89 (2005).

11. Balch CM, Gershenwald JE, Soong SJ, Thompson JF. Update on the melanoma staging system: the importance of sentinel node staging and primary tumor mitotic rate. J. Surg. Oncol. 104(4), 379-385 (2011).

12. Ariyan C, Brady MS, Gönen M, Busam K, Coit D. Positive nonsentinel node status predicts mortality in patients with cutaneous melanoma. Ann. Surg. Oncol. 16(1), 186-190 (2009).

13. de Macedo MP, Franke V, Duprat Neto JP et al. Validation of a nomogram for non-sentinel node positivity in melanoma patients, and its clinical implications: a Brazilian-Dutch study. Ann. Surg. Oncol.26(2), 395-405 (2019).

14. Murali R, Desilva C, Thompson JF, Scolyer RA. Non-sentinel node risk score (N-SNORE): a scoring system for accurately stratifying risk of non-sentinel node positivity in patients with cutaneous melanoma with positive sentinel lymph nodes. J. Clin. Oncol. 28(29), 4441-4449 (2010).

15. Caracò C, Borgognoni L, Caliendo V et al. Prediction of non-sentinel node status in patients with melanoma and positive sentinel node biopsy: an Italian Melanoma Intergroup (IMI) Study. Ann. Surg. Oncol. 25(1), 271-279 (2017).

16. Van Akkooi ACJ, Verhoef C, Eggermont AMM. Importance of tumor load in the sentinel node in melanoma: clinical dilemmas. Nat. Rev. Clin. Oncol. 7(8), 446-454 (2010).

17. Leiter U, Stadler R, Mauch C et al. Complete lymph node dissection versus no dissection in patients with sentinel lymph node biopsy positive melanoma (DeCOG-SLT): a multicentre, randomised, Phase III trial. Lancet Oncol. 17(6), 757-767 (2016).

-. A second randomised controlled trial that came to the conclusion that immediate CLND is not of benefit.

18. Bamboat ZM, Konstantinidis IT, Kuk D, Ariyan CE, Brady MS, Coit DG. Observation after a positive sentinel lymph node biopsy in patients with melanoma. Ann. Surg. Oncol. 21(9), 3117-3123 (2014).

19. Spillane A, McCormack C, Howle J et al. Cancer Council Australia Cancer Guidelines Wiki (2018). http://wiki.cancer.org.au

20. Coit DG, Thomson JA, Albertini MR et al. National Comprehensive Cancer Network. Cutaneous melanoma version 2.2019 (2019). http://jnccn.org/view/journals/jnccn/17/4/article-p367.xml.

21. Grünhagen DJ, van der Veldt AAM, Keilholz U et al. Risk stratification of sentinel node-positive melanoma patients defines surgical management and adjuvant therapy treatment considerations. Eur. J. Cancer 96, 25-33 (2018).

22. Wong SL, Faries MB, Kennedy EB et al. Sentinel lymph node biopsy and management of regional lymph nodes in melanoma: American Society of Clinical Oncology and Society of Surgical Oncology Clinical Practice guideline update. J. Clin. Oncol.36(4), 399-413 (2017).

23. Masoud SJ, Perone JA, Farrow NE, Mosca PJ, Tyler DS, Beasley GM. Sentinel lymph node biopsy and completion lymph node dissection for melanoma. Curr. Treat. Options Oncol. 19(11) (2018).

24. Kwak M, Farrow NE, Salama AKS et al. Updates in adjuvant systemic therapy for melanoma. J. Surg. Oncol. 119(2), 222-231 (2019). 